expression and restores sterol sensing (Figure 1). Upon fasting, Insig-2a levels rise, suppressing lipogenesis in a sterol-dependent fashion. Insulin cycling might be critical for the prevention of excessive lipogenesis. It creates a brief window during refeeding when Insig levels begin to decline and there is insufficient Insig-1 to suppress lipogenesis. However, with induction of Insig-1, lipogenesis is held in check. Thus, a wild-type refed animal "overshoots" its rate of lipogenesis (7). In contrast, transgenic mice overexpressing Insig-1 effectively abolish the cycling of Insig-1 expression and therefore do not overshoot lipogenesis upon refeeding (Figure 1). This leads to a dramatic $80 \%$ reduction in plasma triglycerides in the refed mice (8).

\section{Potential implications for insulin resistance}

With chronic hyperinsulinemia, the normally converse relationship between lipogenesis and gluconeogenesis can be disrupted (16). This occurs in models of leptin deficiency (e.g., the leptin ${ }^{\mathrm{ob}}$ mutation or congenital lipodystrophy) or leptin resistance. Deletion of IRS-2 leads to leptin resistance (17), suggesting a convergence between the leptin and insulin signaling pathways. Leptin resistance boosts lipogenesis in the liver through increased SREBP-1c. Deletion of SREBP-1c reduces the rate of lipogenesis of leptin-deficient animals but does not reverse insulin resistance, hence other aspects of leptin signaling influence insulin signaling (18).
The focus of the Insig story will likely turn to Insig-2a. Is Insig-2a affected by leptin deficiency or leptin resistance? What happens to Insig-2a under conditions of chronic hyperinsulinemia? We hope to learn why two closely related proteins are oppositely regulated by insulin.

Address correspondence to: Alan D. Attie, Department of Biochemistry, University of Wisconsin-Madison, 433 Babcock Drive, Madison, Wisconsin 53706, USA. Phone: (608) 262-1372; Fax: (608) 263-9609; E-mail: attie@biochem.wisc.edu.

1. Tontonoz, P., Kim, J.B., Graves, R.A., and Spiegelman, B.M. 1993. ADD1: a novel helix-loop-helix transcription factor associated with adipocyte determination and differentiation. Mol. Cell. Biol. 13:4753-4759.

2. Yokoyama, C., et al. 1993. SREBP-1, a basic-helixloop-helix-leucine zipper protein that controls transcription of the low density lipoprotein receptor gene. Cell. 75:187-197.

3. Horton, J.D., Goldstein, J.L., and Brown, M.S. 2002 SREBPS: activators of the complete program of cholesterol and fatty acid synthesis in the liver. J. Clin. Invest. 109:1125-1131. doi:10.1172/JCI200215593.

4. Hua, X., Sakai, J., Brown, M.S., and Goldstein, J.L. 1996. Regulated cleavage of sterol regulatory element binding proteins requires sequences on both sides of the endoplasmic reticulum membrane. J. Biol. Chem. 271:10379-10384.

5. Yang, T., Goldstein, J.L., and Brown, M.S. 2000. Overexpression of membrane domain of SCAP prevents sterols from inhibiting SCAP.SREBP exit from endoplasmic reticulum. J. Biol. Chem. 275:29881-29886.

6. Yang, T., et al. 2002. Crucial step in cholesterol homeostasis: sterols promote binding of SCAP to INSIG-1, a membrane protein that facilitates retention of SREBPs in ER. Cell. 110:489-500.

7. Yabe, D., Brown, M.S., and Goldstein, J.L. 2002 Insig-2, a second endoplasmic reticulum protein that binds SCAP and blocks export of sterol regulatory element-binding proteins. Proc. Natl. Acad. Sci. U. S. A. 99:12753-12758.

8. Engelking, L.J., et al. 2004. Overexpression of Insig-1 in the livers of transgenic mice inhibits SREBP processing and reduces insulin-stimulated lipogenesis. J. Clin. Invest. 113:1168-1175. doi:10.1172/JCI200420978.

9. Repa, J.J., et al. 2000. Regulation of mouse sterol regulatory element-binding protein-1c gene (SREBP-1c) by oxysterol receptors, LXRalpha and LXRbeta. Genes Dev. 14:2819-2830.

10. Foretz, M., et al. 1999. ADD1/SREBP-1c is required in the activation of hepatic lipogenic gene expression by glucose. Mol. Cell. Biol. 19:3760-3768.

11. Shimomura, I., et al. 1999. Insulin selectively increases SREBP-1c mRNA in the livers of rats with streptozotocin-induced diabetes. Proc. Natl. Acad. Sci. U. S. A. 96:13656-13661.

12. Shimano, H., et al. 1997. Isoform 1c of sterol regulatory element binding protein is less active than isoform 1a in livers of transgenic mice and in cultured cells. J. Clin. Invest. 99:846-854.

13. Liang, G., et al. 2002. Diminished hepatic response to fasting/refeeding and liver $\mathrm{X}$ receptor agonists in mice with selective deficiency of sterol regulatory elementbinding protein-1c. J. Biol. Chem. 277:9520-9528.

14. Zhang, J., et al. 2001. Insulin inhibits transcription of IRS-2 gene in rat liver through an insulin response element (IRE) that resembles IREs of other insulin-repressed genes. Proc. Natl. Acad. Sci. U. S. A. 98:3756-3761.

15. Puigserver, P., et al. 2003. Insulin-regulated hepatic gluconeogenesis through FOXO1-PGC-1alpha interaction. Nature. 423:550-555.

16. Shimomura, I., et al. 2000. Decreased IRS-2 and increased SREBP-1c lead to mixed insulin resistance and sensitivity in livers of lipodystrophic and ob/ob mice. Mol. Cell. 6:77-86.

17. Tobe, K., et al. 2001. Increased expression of the sterol regulatory element-binding protein-1 gene in insulin receptor substrate-2/- mouse liver. J. Biol. Chem. 276:38337-38340.

18. Yahagi, N., et al. 2002. Absence of sterol regulatory element-binding protein-1 (SREBP-1) ameliorates fatty livers but not obesity or insulin resistance in Lep $^{\mathrm{ob}} /$ Lep $^{\mathrm{ob}}$ mice. J. Biol. Chem. 277:19353-19357.

\title{
Experimental autoimmune hearing loss
}

\author{
Peter Billings
}

Division of Otolaryngology—Head and Neck Surgery, University of California, San Diego, and Research Service of the Department of Veterans Affairs, San Diego, California, USA.

\begin{abstract}
Understanding of autoimmune sensorineural hearing loss (ASNHL) has been hindered by the inaccessibility of the inner ear to biopsy and the lack of workable animal models. A report in this issue of the JCI describes a mouse model of $\mathrm{CD}^{+} \mathrm{T}$ cell-mediated ASNHL induced by immunization with peptides from the inner ear-specific proteins cochlin and $\beta$-tectorin (see the related article beginning on page 1210).
\end{abstract}

\author{
Nonstandard abbreviations used: antigen (Ag); \\ autoimmune inner ear disease (AIED); autoimmune \\ sensorineural hearing loss (ASNHL); inner ear (IE); \\ sensorineural hearing loss (SNHL). \\ Conflict of interest: The author has declared that no \\ conflict of interest exists. \\ Citation for this article: \\ J. Clin. Invest. 113:1114-1117 (2004). \\ doi:10.1172/JCI200421632.
}

The inner ear (IE), like most other specialized tissues and organs, can become the target of an autoimmune attack. Sensorineural hearing loss (SNHL) is often an early, although presumably secondary, complication of various non-organ-specific autoimmune diseases; however, the IE can also represent the primary focus of a unique disease entity, autoimmune IE disease (AIED) (1). Fortunately the disease is rare, but the small population size of affected individuals and the inaccessibility of the IE during an acute attack have hindered progress in our understanding of the etiology, diagnosis, and treatment of this disease. AIED is diagnosed by exclusion of other disorders that mimic it. The hearing loss is typically bilateral, asymmetric, and fluctuating and deteriorates rapidly over weeks or months; balance and equilibrium may or may not be affected. Diagnosis of AIED is tentatively confirmed if there is a positive response to trial corticosteroid 


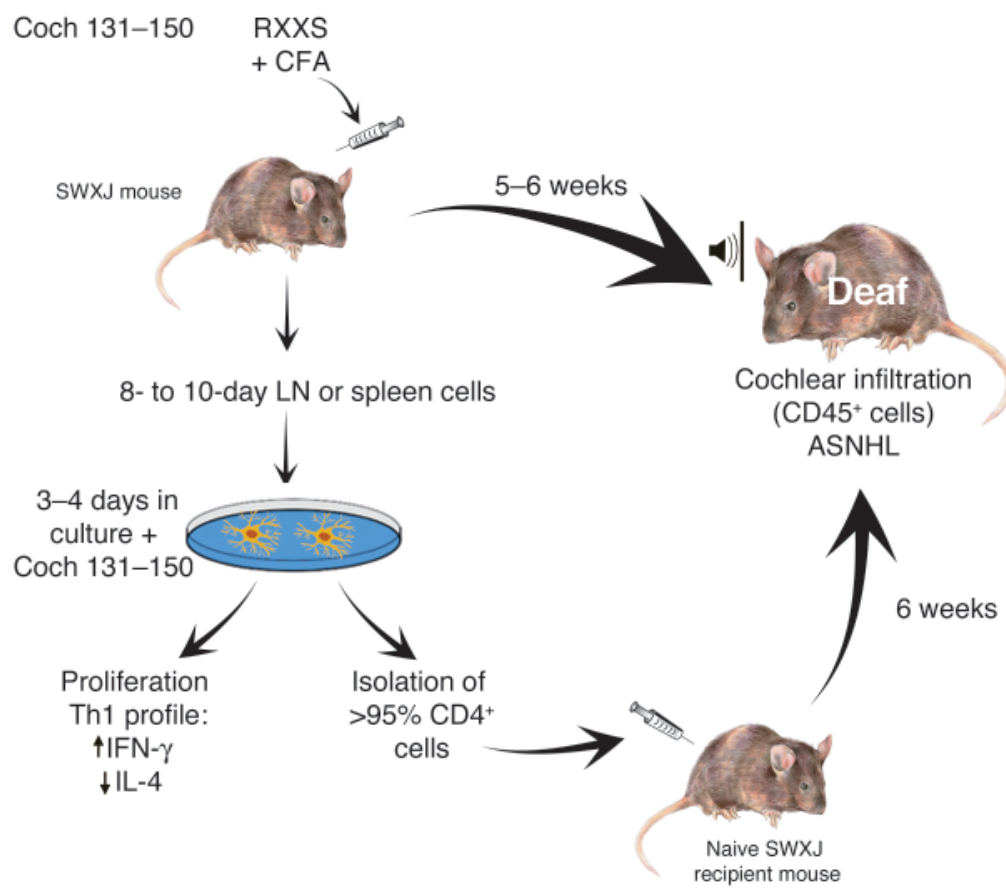

therapy, although some individuals experience spontaneous recovery. Therapy must be initiated rapidly, since unchecked inflammatory damage to the fragile and irreplaceable sensory structures of the IE may easily lead to irreversible SNHL. On the other hand, systemic immunosuppressive drugs may increase susceptibility to infection, and steroids may induce water retention; hence the urgent need for better supporting diagnostic procedures for idiopathic SNHL, as well as more locally focused treatments. In general, we need a great deal more fundamental information about AIED before substantial progress can be made in its diagnosis and treatment.

Current aids in the diagnosis of AIED Understanding of AIED has been hampered most by the inability to monitor events occurring within the bony confines of the IE during the course of the disease. We do not know what cells, in what proportions and with what antigen (Ag) specificities, are involved; nor do we know the relative role(s), if any, of the array of associated autoantibodies. The few human temporal bones derive from lateor end-stage AIED and show a marked osteoneogenic response to chronic IE inflammation (2). Given the inaccessibility of this area for biopsy, noninvasive procedures have been used for diagnostic testing. One such test measures recall responses of T cells to $\operatorname{IE~} \mathrm{Ag}(3)$, but it is inherently difficult and not readily available, and it only measures the levels of circulating, not nodal or IE-sequestered, lymphocytes. Tests for autoantibodies have identified multiple Ag's associated with AIED, but there remains considerable controversy in the literature over which Ag's are recognized and by what fraction of patients. Differences in disease-associated Ag's reported between laboratories may derive in part from the use of different Ag sources, methods of $\mathrm{Ag}$ extraction, immunoblotting procedures, and relative molecular mass standards, as well as patient-selection criteria (see refs. 4 and 5), but as yet, there has been no exchange of sera between laboratories using different assays. Even with a single assay, identification of different Ag's may reflect useful information regarding patient heterogeneity, e.g., disease stage, severity, activity, prognosis, susceptibility to intervention, etc.; however, confirmation must await much larger patient study groups. To date, no single Ag has been shown to be recognized by a high proportion of patient but not control sera. A battery of such Ag's may eventually emerge. Of the Ag's that have been biochemically characterized, most have been ubiquitous or common to non-IE-specific tissues and have therefore lacked logical or compelling association with pathology focused on the IE (6-8). When tested as immunogens with strong adjuvants in

\section{Figure 1}

Systemic immunization of autoimmune-prone SWXJ mice with Coch 131-150, an RXXS peptide from the abundant IE extracellular protein cochlin, induced CD45+ cell cochlear infiltration and ASNHL in mice within 5-6 weeks. Lymph node (LN) or spleen cells placed in culture 8-10 days after immunization proliferated, as evidenced by ${ }^{3} \mathrm{H}$-thymidine incorporation, and showed a Th1 cytokine profile (high levels of IFN- $\gamma$ and low levels of IL-4 production) after restimulation in vitro with Coch 131-150. Flow cytometry confirmed preferential reactivation of $\mathrm{CD} 4^{+} \mathrm{T}$ cells. After restimulation in vitro with peptide, the total population or the $\mathrm{CD}^{+}{ }^{+}$-enriched population (>95\%) of cells induced ASNHL 6 weeks after passive transfer to naive, irradiated, histocompatible recipient mice. The same results were obtained after priming, restimulation, and transfer of $\mathrm{CD} 4^{+} \mathrm{T}$ cells specific for a KXXS peptide from a second IE protein, $\beta$-tectorin 71-90, but not with ovalbumin as $\mathrm{Ag}(16)$.

animals, the better-characterized putative autoantigens, such as collagen II, Hsp70, and myelin $\mathrm{P}_{0}$, have given inconsistent results or failed to elicit SNHL or IE inflammatory infiltration (6-8).

Animal models of autoimmune SNHL Attempts to develop an animal model of AIED by immunization with relatively crude IE homogenates have met with limited success; either some animals were unresponsive or the model was underdeveloped and therefore unsuitable for determining the mechanism(s) of IE impairment, the immunologically active components involved, and their location (9-11). Progress is slowly being made in fractionation of the IE immunogen (12), but active fractions may still contain numerous components. Reproducing the exact model in other laboratories and overcoming animal-to-animal variation may be difficult until effective recombinant Ag's are available. Experimental autoimmune SNHL (ASNHL) has been passively transferred in some animal models by $\mathrm{T}$ cell transfer $(10,13)$, demonstrating the critical role of $\mathrm{T}$ cells in the induction of ASNHL. On the other hand, there is also precedent for a critical role of autoantibodies in ASNHL. Administration of the IgG1 mAb KHRI-3 induced SNHL and loss of hair cells (14). KHRI-3 reacts with a glycoprotein $\mathrm{Ag}$ homologous to the human choline transporter CTL-2 
(15), expressed on supporting cells in the organ of Corti. Patient Ab's or mAb's against other Ag's or better epitopes of the same Ag may be effective at more physiologically relevant titers. Thus, there is ample evidence to support that experimental ASNHL is either T cell- or autoantibody-mediated, and human AIED may be mediated by either mechanism, or both, with some patients polarized into $\mathrm{T}$ cell-mediated disease and others into autoantibody-mediated disease and reactive to different Ag's. A reproducible, easyto-establish animal model of AIED would provide a very useful system in which to address questions regarding the early progression and final resolution of an IE autoimmune attack, how and which cells access the IE, and what constitutes a potent IE autoantigen.

\section{Induction of ASNHL by immunization with peptides from IE proteins}

Previous models of experimental ASNHL established by immunization with a complex mixture of IE proteins have been highly erratic. This is understandable, since only some of the proteins were IEspecific, present in immunogenic quantity, and able to sufficiently break self-tolerance in such a manner as to affect hearing in even a fraction of the recipients. In this issue of the JCI, Solares, Tuohy, and colleagues (16) present a novel, straightforward approach to the development of experimental animal models of AIED. Reasoning that the best candidate Ag's would be abundant, IEspecific proteins, the authors restricted their study to just two such proteins, cochlin and $\beta$-tectorin. Since neither protein was available in purified or recombinant form, they immunized autoimmuneprone SWXJ mice with peptides selected from the known sequences of these two extracellular proteins. The peptides contained a lysine or arginine residue separated from a serine by two amino acids, i.e., KXXS or RXXS. Tuohy and coworkers have previously shown that MHC class II molecules on APCs in the SWXJ hybrid mouse strain and in the parental SJL/J and SWR/J mice bind and present peptides bearing the KXXS or RXXS motif (17). Furthermore, in models of other autoimmune diseases, immunization of these mice with KXXS or RXXS peptides derived from known major autoantigens was shown to mediate the corresponding organ-specific autoimmune disease (17).
In the present experiments immunizing with cochlin (Figure 1) and $\beta$-tectorin peptides emulsified with CFA, not all peptides with this motif stimulated an immune response as monitored by $\mathrm{T}$ cell proliferation upon re-exposure of lymph node cells to peptide in vitro (16). However, two peptides, Coch 131-150, containing RXXS, and $\beta$-tectorin 71-90, containing KXXS, stimulated strong recall responses. The lymph node cells that responded were shown by flow cytometry to be $\mathrm{CD}^{+} \mathrm{T}$ cells and produced high IFN- $\gamma$ and low IL- 4 levels characteristic of a Th1 response. The recall response to Coch 131-150 was restricted to I-A ${ }^{\text {s }}$ occurring in both SWXJ hybrid (I-A $\mathrm{A}^{\mathrm{q}, \mathrm{s}}$ ) mice and the SJL/J $\left(I-A^{s}\right)$ parental strain, while the response to $\beta$-tectorin 71-90 was restricted to $\mathrm{I}-\mathrm{A}^{\mathrm{q}}$ occurring in the hybrid and the SWR/J (I-Aq) parent. Most importantly, mice immunized with either peptide demonstrated significant broad-frequency SNHL 5 weeks after immunization. Infiltration of the cochlea by $\mathrm{CD} 45^{+}$leukocytes was coincident with induction of experimental ASNHL after immunization with Coch 131-150. ASNHL was passively transferred to irradiated naive mice by transfer of lymph node cells or spleen $\mathrm{CD}^{+} \mathrm{T}$ cells, which had been removed from donor mice 10 days after immunization and restimulated in vitro with Coch 131-150 or $\beta$-tectorin 71-90.

The ASNHL induced by immunization with Coch 131-150 or $\beta$-tectorin 71-90 in this model demonstrates that autoimmune responses to quite different cochlear Ag's may adversely affect the IE (16). It is tempting to speculate that multiple autoantigens, perhaps including cochlin and $\beta$-tectorin, are also implicated in AIED in humans; however, it is not yet clear how accurately this model reflects events occurring in the spontaneous idiopathic disease or how heterogeneous AIED is with respect to autoantigens. Multiple autoantigens would certainly be consistent with the diverse array of putative autoantibodies reported to be associated with AIED (see refs. 4 and 5). Autoantibodies to cochlin have been identified in individuals with AIED (18), but a prior study reported a correlation between AIED and these autoantibodies in only $10 \%$ of patients ( 4 of 40 ; ref. 5 ). If cochlin and $\beta$-tectorin are involved in AIED, are they functioning as $\mathrm{T}$ cell or $\mathrm{B}$ cell Ag's? Could the present model be extended to almost any IE-specific Ag, or, as is the case with the extracellular Ag's cochlin and $\beta$-tectorin, is induction of ASNHL dependent on a highly abundant target Ag that is readily accessibility to $\mathrm{T}$ cells? Peptides from other IE proteins and non-IE-specific proteins will undoubtedly be tested in this model. With a list of autoantigens capable of inducing experimental ASNHL disease in animal models, AIED patients could be screened for $T$ and $B$ cell reactivities against those same Ag's to determine whether these Ag's are involved in the human disease. A number of questions still remain. What are the exact nature and kinetics of the IE damage resulting from immunization with IE Ag's; do they differ with different Ag's? Is the damage reversible? Is pathology dependent on the identity and location of the target Ag, or is it in part a bystander effect? What cells are infiltrating the cochlea? Is it mainly $\mathrm{CD}^{+}$cells? What cells do they recruit? How do cells and Ab's cross the so-called blood-labyrinth barrier, analogous to the blood-brain barrier in the CNS? These questions, and many others as yet unasked, may be amenable to investigation by many laboratories using this simple and reproducible animal model. Questions regarding the etiology and self-perpetuation of spontaneous AIED may not be as easily addressed in a transient model, where the initiating agent or event is artificial and apparent; however, we can expect that this model will provide many answers and lead to better, more representative, and workable models of AIED.

Address correspondence to: Peter Billings, Division of Otolaryngology-Head and Neck Surgery, University of California, San Diego, and Research Service of the Department of Veterans Affairs, $3350 \mathrm{La}$ Jolla Village Drive, San Diego, California 92161, USA. Phone: (858) 552-8585 ext. 6115; Fax: (858) 552-7436; E-mail: pbillings@ucsd.edu.

1. Harris, J.P., Moscicki, R.A., and Hughes, G.B. 1997. Immunologic disorders of the inner ear. In Clinical otology. G.B. Hughes and M.L. Pensak, editors. Thieme Medical Publishers. New York, New York, USA. 381-391.

2. Keithley, E.M., Chen, M.-C., and Linthicum, F. 1998. Clinical diagnoses associated with histologic findings of fibrotic tissue and new bone in the inner ear. Laryngoscope. 108:87-91.

3. Hughes, G.B., Moscicki, R., Barna, B.P., and San Martin, J.E. 1994. Laboratory diagnosis of immune inner ear disease. Am. J. Otol. 15:198-202.

4. García Berrocal, J.R., Ramírez-Camacho, R., Arellano, B., and Vargas, J.A. 2002. Validity of the Western blot immunoassay for heat shock protein-70 in 
associated and isolated immunorelated inner ear disease. Laryngoscope. 112:304-309.

5. Cao, M.Y., Deggouj, N., Gersdorff, M., and Tomasi, J.-P. 1996. Guinea pig inner ear antigens: extraction and application to the study of human autoimmune inner ear disease. Laryngoscope. 106:207-212.

6. Harris, J.P., Woolf, N.K., and Ryan, A.F. 1986. A reexamination of experimental type II collagen autoimmunity: middle and inner ear morphology and function. Ann. Otol. Rhinol. Laryngol. 95:176-180.

7. Billings, P.B., Shin, S.-O., and Harris, J.P. 1998. Assessing the role of anti-hsp70 in cochlear function. Hear. Res. 126:210-212.

8. Boulassel, M.R., et al. 2001. No evidence of auditory dysfunction in guinea pigs immunized with myelin P0 protein. Hear. Res. 152:10-16.
9. Harris, J.P. 1987. Experimental autoimmune sensorineural hearing loss. Laryngoscope. 97:63-76.

10. Ikezono, T., et al. 2000. Passive transfer of experimental labyrinthitis. Audiol. Neurootol. 5:292-299.

11. Bouman, H., et al. 2000. Experimental autoimmune inner ear disease: an electrocochleographic and histophysiologic study. Ann. Otol. Rhinol. Laryngol. 109:457-466.

12. Tomiyama, S. 2002. Experimental autoimmune labyrinthitis: assessment of molecular size of autoantigens in fractions of inner ear proteins eluted on the Mini Whole Gel Eluter. Acta Otolaryngol. 122:692-697.

13. Gloddek, B., Gloddek, J., and Arnold, W. 1999. A rat T-cell line that mediates autoimmune disease of the inner ear in the Lewis rat. ORLJ. Otorbinolaryngol. Relat. Spec. 61:181-187.

14. Nair, T.S., et al. 1995. Monoclonal antibody induced hearing loss. Hear. Res. 83:101-113.

15. Nair, T.S., et al. 2002. Evidence that the inner ear supporting cell antigen (IESCA) is CTL2, a member of the choline transporter-like family. Assoc. Res. Otolaryngol. 256 (Abstr.). http://www.aro.org/archives/ 2002/2002258.html.

16. Solares, C.A., et al. 2004. Murine autoimmune hearing loss mediated by $\mathrm{CD}^{+} \mathrm{T}$ cells specific for inner ear peptides. J. Clin. Invest. 113:1210-1217. doi:10.1172/JCI200418195.

17. Jane-wit, D., et al. 2002. A novel class II-binding motif selects peptides that mediate organ-specific autoimmune disease in SWXJ, SJL/J, and SWR/J mice. J. Immunol. 169:6507-6514.

18. Boulassel, M.-R., Tomasi, J.-P., Deggouj, N., and Gersdorff, M. 2001. COCH5B2 is a target antigen of anti-inner ear antibodies in autoimmune inner ear diseases. Otol. Neurotol. 22:614-618. 